\title{
FT-NIR, MicroNIR and LED-MicroNIR for detection of adulteration in palm oil via PLS and LDA
}

\begin{abstract}
Chemometrics analysis was performed to compare the performance of FT-NIR, MicroNIR and LED-NIR for detection of adulteration in palm oil. FT-NIR has a high spectral resolution and signal-to-noise ratio, but MicroNIR is more light weight and suitable for on-site application. The feasibility of LED to replace the conventional halogen tungsten light source in MicroNIR has been discussed in this paper. The wavelength of LEDs was based on the variable selection method, CARS, and the results were in good agreement with the $\mathrm{C}-\mathrm{H}$ and $\mathrm{O}-\mathrm{H}$ bond interaction displayed in the observed NIR spectrum. The advantages of using LED instead of a halogen tungsten light source are cost effectiveness, low power consumption and reduced number of variables. Different pretreatment approaches has been applied to the spectral data acquired to investigate the performance of preprocess to the result of chemometrics. Quantitative analysis was performed using partial least square (PLS) algorithms with the linear regression method. The best correlation coefficient, (R2), reported using FTNIR was 0.99 with RMSEC and RMSEP values less than 1, indicating that the spread of calibration and prediction data was small. The LDA result showed that LED-NIR outperforms FT-NIR and MicroNIR with a sensitivity of 1.00 and a specificity of 0.9333 .
\end{abstract}

Keyword: FT-NIR; MicroNIR; LED-MicroNIR; Adulteration; Palm oil 
